\title{
Data Communications in High Energy Physics
}

\author{
M. G. N. Hine, CERN, Geneva
}

The role of data communications as part of the general technology of high energy physics has evolved rapidly, because of both the nature of the subject and the way research is carried out. This evolution can be paralleled in some other subjects, but HEP gives one of the more striking examples and may prefigure the way other branches of science will develop in future.

Around 1950, a considerable number of universities and other research institutes started building particle accelerators in the $\mathrm{GeV}$ range of energy to provide their physics departments with the tools which Berkeley and Brookhaven had shown to be essential for research into elementary particles, and which clearly outclassed cosmic rays in almost all respects. Although a few laboratories such as CERN were planned as cooperative efforts to serve large communities of physicists based on universities spread over a country or a continent, it was only in the 1960s that it became clear that the typical university could no longer afford to run its own accelerator and keep up with the field. Moreover, all experimenters would have to split their time, in often uncomfortable ways, between their own departments and a very few central accelerator laboratories. Nuclear physics, as it was still called, became a science like Egyptology; one could no longer have a pyramid on the campus, but would have to work abroad.

Fortunately, at that time the development of jet travel made it easier for people to spend short stays at other laboratories, and the main bulk of experimental data was on bubble chamber film, which could be transferred physically, and also allowed the analysis phase of experiments to be carried out at the physicists' home laboratories in a rather independent way. Further, the experimental groups were usually quite small, and internal communications could be managed by meetings, telephone calls, and the exchange of paper.

By 1975, with the arrival of the SPS at CERN, the size of experiments and the number of collaborators in each had grown considerably, and experiments using electronic detectors and on-line computers were producing enormous amounts of digital data at the accelerator. Groups of 20-50 physicists from 5-10 different labs. were the rule for the big experiments, and these often produced hundreds of magnetic tapes, or more, per year, which required complex analysis programmes running on large computers. Both because of the com- puting power required and because it was felt as a matter of policy that physics analysis should be done mainly in the home institutes, these computers were of many types, requiring specially adapted programmes subject to frequent change. This distribution of computing had been true also for bubble chamber analysis, but there the programmes were much more stable and independent of the particular experiment being analysed, so their writing and distribution was a simpler matter.

Today this evolution, both in the 'sociology' and in the technique of experiments, is continuing, and will do so for the next generation of accelerators now located only in CERN and DESY in Europe. LEP will be equipped with only four experiments at the start, and the remainder of CERN's facilities will be considerably curtailed for budget reasons, including the stopping of the ISR. The continuing concentration of people into large groups is shown in the early Letters of Intent for the first LEP experiments. These were signed by around 20 institutes each, and the teams by now contain $\mathbf{2 0 0}$ or more members. The detectors and the associated amount of data in each event are larger than in any earlier experiments. The data capture systems, often involving large clusters of microprocessors, will be an order of magnitude more complicated than those of today. Despite the probable lower event rates in LEP experiments than in the SPS, the total quantity of data and the computer power needed to analyse the physics will also be larger than at present.

\section{Development of Data Communications}

This picture of the human and technical sides of high energy experiments shows clearly the growing need for better communications between people, between people and their equipment and computers, and between computers themselves. Fortunately, or perhaps as a consequence of more general trends in industrial society, of which high energy physics is a part, the techniques for better communication were evolving on about the same time scale. Laboratories in some countries, particularly in the UK, began to be equipped with remote job entry stations linked by telephone lines to large service computers, well adapted to the HEP programmes; inside the main laboratories data collection computers were linked to central machines, and users began to get terminal access to replace the ubiquitous punched cards.
The last ten years have seen the arrival of specialized long distance data transmission networks, with associated communities of scientific users who are able to collaborate in sharing computers, passing messages, documents, computer programmes and data between themselves with essentially no delay. The leader in this new field was the US ARPA packet switched network, which has been followed by public networks such as Telenet in the USA, and more recently by national PTT-organized networks in Europe, such as Transpac in France, Datex-P in the Federal Republic of Germany and PSS in the UK. These networks give the equivalent of the dial-up telephone service for data transmission of high quality, at speeds typically up to 10 $\mathrm{kbit} / \mathrm{s}$. This is sufficient for a large amount of the traffic between people and computers, e.g. for terminals and line printers, and for transfer of moderate size files between computers, but not for large quantities of raw physics data on magnetic tapes. These still have to be sent by post or carried by hand.

For the high energy physicists, the first country in Europe to follow this development was the UK, where the early network of remote job stations in all the universities connected to the computer centres at Rutherford and Daresbury Laboratories has been progressively replaced by a private packet network, SERCNET, using the same techniques and protocols as the new public networks. This network is extended to CERN via a leased PTT line, so that UK physicists at CERN can use the computers at Rutherford or exchange files and messages with colleagues in any of the UK laboratories in their experiments. A similar arrangement exists for DESY. Inside CERN there is a megabit/s packet net, CERNET, which interconnects some 70 computers on the site, and the accelerators today have control systems based on similar networks to connect all the machine components to the control rooms. DESY and other large laboratories have similar on-site network facilities where they have computers in different buildings working together, and they have been setting up some external connections to other laboratories.

Public data services in Europe are rapidly becoming available in all countries, with international connections, so that by the end of 1983 it should be possible for almost all physics institutes in the CERN and DESY programmes to have the same data transmission services as their colleagues in the UK have today. This positive forecast must be qualified, however, by saying that this is equivalent to offering an international telephone service to physicists in several countries: they can dial up their colleagues, but if they don't speak the same language, they cannot usefully communicate information. The international agreements on data transmission go as far as ensuring compati- 
bility for sending streams of bits from one place to another, but not of allowing computers of different types and makes to interpret these bits as questions or files etc. Progress on this problem of standards will come in some years time, but meanwhile special measures to translate the different "higher level protocols" between incompatible computer systems have to be worked out and provided inside each particular community of users concerned.

The UK physicists do not have this problem, because the SERC network authority, which has Government funding, has been able to enforce a single set of protocols on all the types of computer attached to the network. There is, however, no corresponding international authority over university computer centres, and the HEP community has therefore started to try to arrange a coordinated approach to the problem with the help of the main HEP centres, through the European Committee for Future Accelerators, ECFA, which has been in the past the discussion centre for physicists on all aspects of HEP in Europe.

In one respect the new public networks cannot provide the same service as can, for example, CERNET inside CERN for the rapid transfer of large amounts of data, e.g. a 100 Megabyte sample of events, or the remote control of a real time system needing more than kilobit/s data rates. The extension of these services to higher speeds, up to $2 \mathrm{Mbit} / \mathrm{s}$, is foreseen by the PTTs, using satellite transmission links between small earth stations located near the users' premises, thus avoiding the need for tying up very expensive high speed ground links. The use of the satellite implicity offers switched services between all users, since the down-coming signals can be received by all stations.

\section{STELLA Experiment}

To test out some of the techniques for getting very low error rates and for exploring the interest of this kind of service for high energy physicists, an experiment, STELLA, was set up in 1978 by CERN, the European Space Agency, and several other laboratories, with the help of the Commission of the European Communities. The experiment involved several small earth stations, at CERN, Rutherford, Pisa, DESY and elsewhere, with the possibility of copying magnetic tapes and large files at average speeds of about $1 / 2 \mathrm{Mbit} / \mathrm{s}$, and with no errors. An extension to the system, now being developed, will use the satellite links to interconnect the main local networks at CERN, Rutherford and Pisa, so as to allow a much wider range of traffic to be handled, and to make the system more like what would be the most probable use of the corresponding PTT services when they become available in 1984. A similar experiment, Universe, based on Stella experience has started operating in the UK with PTT and industry participation.
Particular Applications in High Energy Physics

In view of the evolution of the European HEP programme and of the technology of experiments and of communications, ECFA set up in 1980 a Working Group to look into the whole area of data handling for the LEP era, and in particular the needs for new methods of data communications and how these needs might be satisfied. The Subgroup which took on this task established the picture of high energy physics and of communications summarized above, and described a range of User Facilities for physicists which would be needed for the LEP experiments and could be provided if good data communications existed ${ }^{1}$ ).
Examples given were:

1. Remote access to the usual physics computing and data-processing functions with return of output to local printers or terminals.

2. Software management: libraries, documentation, distribution of updates, archiving.

3. Remote monitoring, testing, and fault diagnosis of equipment in experiments.

4. Computer aided design for electronics and mechanical equipment.

5. Tools for collaborative development of software, and for distributed computing in general.

6. Systems for producing, storing, and distributing text of reports, minutes, etc.

\section{Twente University of Technology}

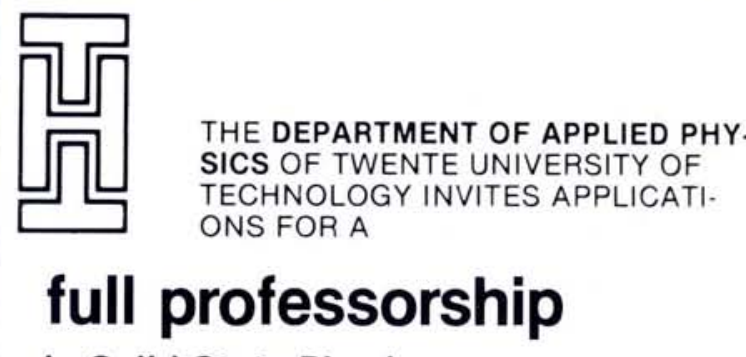

in Solid State Physics

The appointee will be the successor to the late professor G.A. Bootsma.

He will lead the Solid State Physics Group. This group comprises five scientific staffmembers, four technicians and five research-associates. The research concentrates on the equilibrium and transport phenomena at, or near, the boun. daries of semiconductors or metals. Fundamen. tal and applied aspects of these phenomena receive equal attention.

\section{Among the duties of the candidate are:}

- continuation of the existing research program of the group

- sharing in the teaching load of the group

- participation in the administration of the group and the department.

A working knowledge of the Dutch language is expected after two years.

The Twente University of Technology is a Federal University. Excellent benefits are provided by the Federal State of the Netherlands. The tenure is at one of the highest civil service ranks.

Applications, included an extensive curriculum vitae, list of publications and several references, should be sent to the chairman of the Selection Committee (within a period of 4 weeks after date of publication):

Professor dr. L.C. van der Marel.

Department of Applied Physics,

Twente University of Technology,

P.O. Box 217, 7500 AE Enschede,

The Netherlands.

Phone 31: (53)-893366.

Requests for further information and/or sug. gestions for suitable candidates can be sent to the same address. 
7. Data bases and information retrieval facilities for scientific and administrative data, library material, planning and schedules.

8. Electronic mail, computer conferencing. In addition to User Facilities like the above, most of which would preferably be maintained and kept coherent for use by the whole community by the main computer centres or central laboratories, the general use of networks would equally offer communications between individual users for their own messages, programme files, test results, and for many distributed applications, on a scale depending mainly on the equipment installed locally.

All these facilities have been found to be important in the design and running of large experiments, in the preparation of reports and papers for publication, and in general communication inside collaborations, by those physicists who already have access to good communication facilities. The current developments in networking should make it possible to offer them to all members of experiment collaborations, and so help to offset the disadvantage now felt by many people in smaller laboratories compared with their colleagues attached to the large or well equipped centres.

The most apparent problems to be solved before such a networked community can operate really effectively are the absence of agreed protocols for user functions like file transfer between different types of computer, and, later on, the present speed limit of $9.6 \mathrm{kbit} / \mathrm{s}$ for international connections. As mentioned above, ECFA is proposing a collaboration between the main interested laboratories to work on medium term solutions to the protocol problem, until internationally agreed standards appear and can be implemented on the computers used by physicists. The programme has two parts, to establish a common way of using the so-called Triple $X$ protocols for terminals on the public networks, and creating a set of translators between the four file transfer protocols most commonly used by the high energy community. These two developments should be completed and tested in the course of 1984 , and are essential contributions to the general use of networks in the ways desired by ECFA. It is clear that there is little in this programme which is specific to high energy physics, and it would benefit all other scientific communities who also have problems of collaboration at a distance.

The need for speeds higher than 9.6 $\mathrm{kbit} / \mathrm{s}$ has been studied practically by several laboratories in the STELLA experiment, in which about 500 magnetic tapes were sent over 18 months between CERN, Rutherford and Pisa for physicists actually running experiments at CERN, or analysing physics data.

The conclusions so far are that the limited type of service offered by STELLA, transfer of full magnetic tapes, is of limited interest as a generally usable way of shipping bulk data, since most of the tapes produced in an experiment are not needed urgently at home, and the cost of using a public data service would be prohibitive. However, from this experience it has also appeared that there are many occasions when large files or small samples of data are needed at short notice in distant laboratories, allowing rapid feedback on the progress of an experiment, keeping people at home up-to-date in the analysis, and helping with urgent diagnosis of malfunctions. The transmission speeds necessary for these applications range between $50 \mathrm{kbit} / \mathrm{s}$ and $1-2 \mathrm{Mbit} / \mathrm{s}$, which are the speeds to be offered by the PTTs from 1984 when the two European satellites equipped for data transmission, ECS2 and Telecom 1, come into service. The use of these services will depend greatly on the tariffs, which are not yet announced by the PTTs. It is possible that it will be felt justifiable to have occasional use of the satellite services between the large computer centres for the type of high priority or emergency transmissions mentioned above. The possibility of using the ground based networks at $48 \mathrm{kbit} / \mathrm{s}$ for international calls that constitute much of this traffic is not excluded, but this does not seem likely for some time to come.

\section{Economics}

Connecting all the high energy laboratories to the new networks, and using them intensively, will of course add to the operating costs of the research groups, unless, as in the UK, the network is provided as a general facility like an accelerator from a different budget. The PTT tariffs vary considerably from one country to another, and there is the usual scandal, as with the telephone: international calls cost several times those inside one country for the same distance. Some estimates have been made by ECFA, on the basis of known tariffs for networks and leased lines, the UK experience in the use of SERCNET, and that of Saclay and others with leased line connections. The general conclusion is that these data communication facilities are as essential to the physicists' work as are the computers themselves, once their possibilities are exploited, and that the costs for typical groups are going to be a small fraction of their travel budgets for working at CERN, going to collaboration meetings, etc.

It is believed that part at least of the cost could be covered by having to travel less: in any case it seems clear that it is well worth while in terms of increased efficiency for the physicists in doing their work and getting their results out quickly.

\section{REFERENCE}

1. Networks for High Energy Physics, an Interim Report on Wide Area Communications,

ECFA/82/60, (CERN, Geneva) 1982.
Eight two- to four-day seminars on advanced optical/electro-optical technology.

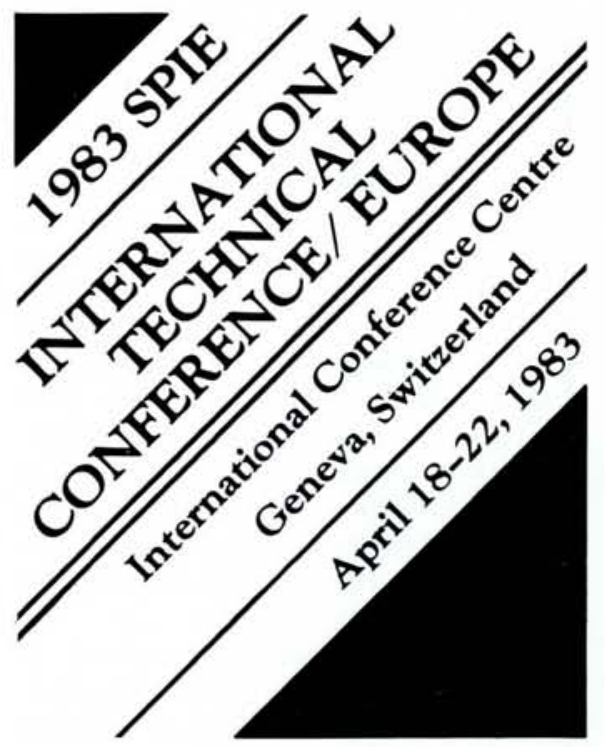

- Advances in Laser Scanning and Recording

- Advanced Infrared Sensor Technology

- Applications of Digital Image Processing

- Industrial Applications of Laser Technology

- Optical System Design, Analysis, and Production

- Thin Film Technologies

- Three-Dimensional Imaging

- New Optical Materials

Approximately 300 papers will be presented, in addition to fourteen tutorials on related topics, and an international instrument display

Sponsored by SPIE-The International Society for Optical Engineering.

COOPERATINGSPONSORS: Association Nationale de la Recherche Technique; Battelle-Geneva Research Centres; Comité Francais d'Optique: European Optical Committee (representing National Optical Committees); European Physical Society: German Society for Applied Optics; IEEE Computer Society; Italian Electronic and Electrotechnica Association; Swiss Federal Institute of Technology in Lausanne; Swiss Society for Optics and Electron Microscopy.

FOR ADDITIONAL PROGRAM AND REGISTRA TION INFORMATION: Contact SPIE, P.O. Box 10 , Bellingham, WA 98227-0010 USA, telephone (206) 676 3290, telex 152556 SEA. In Europe, contact the European Physical Society, P.O. Box 69, CH-1213 Petit-Lancy 2, Switzerland, telephone (022) 93-11-30, telex 23-455 (alarm ch). FOR EXHIBIT INFORMATION: Contact the U.S. offices of SPIE. In Europe, contact Vicki BASTIEN rue du Poulain, Villey-St. Etienne, 54200 Toul, France, telephone (8) 343-9461, telex 850078 (subscriber No. 85). 